\title{
A Novel Remote Sensing Image Change Detection Algorithm based on Game Theory Analysis and Hierarchical Fuzzy Clustering
}

\author{
Xinyu Zhang ${ }^{1,}$, Xuan Zhuang ${ }^{2}$ and Hang $\mathrm{Ji}^{3}$ \\ ${ }^{1}$ Graduate School of Art and Science (Statistics), Columbia University, New York, USA; \\ ${ }^{2}$ School of Electronic Engineering, Xidian University, Xi'an, China; \\ ${ }^{3}$ Dongling School of Economics and Management, University of Science and Technology Beijing, \\ Beijing, China. \\ azhangxinyuny@sina.com
}

Keywords: Remote Sensing, Chang Detection, Game Theory, Fuzzy Clustering, Algorithm.

\begin{abstract}
A novel remote sensing image change detection algorithm based on game theory analysis and hierarchical fuzzy clustering is proposed in this paper. This technique integrates the Nash game theory framework and the hierarchical structured clustering approach. Characteristics of the image restoration and segmentation are analyzed to serve as the pre-processing step with the optimal tactical balance. With the deepening of the hierarchy, distributed clusters inside tightness degree gets less, at the same level of processing conforms to close conditions in current layer with sub-manifold merge, therefore, we choose hierarchical fuzzy clustering to finalize the change detection task. Experimental result confirms the effectiveness and feasibility of the proposed methodology. It increases the overall accuracy and robustness of the change detection processes compared with other approaches.
\end{abstract}

\section{Introduction}

In recent years, aviation remote sensing technology has achieved rapid development. Re-railing ability and stability of the satellite remote sensing series consistent sensor parameters and operation plan have been enhanced, the repetition of the same area implements the continuous observation, acquired long phase, multi-scale in remote sensing image detailedly records time and space dynamic change process of all kinds of features on the earth's surface and the trajectory. Under this technique background, the change detection (CD) has been proposed to access the difference of two registered remote sensed multi-spectral images acquired in the same geographical area during different targeted time. According to literature review, the state-of-the-art CD algorithms could be roughly separated into two categories. (1) Change detection at pixel level. Pixel level change detection uses change before and after the first image to structure difference image and then adopts unit change information extraction approaches to find the optimal threshold through the difference image binarization. The corresponding algorithms are statistical distribution based, wavelet based and Markov random field based models. (2) Object level change detection. With the constant improvement of the SAR image resolution, pixel level based change detection is more and more difficult to meet the needs of the data processing. Compared with the traditional medium and low spatial resolution images, high resolution images of the space information becomes richer, the details of the object target information express are more clearly and the relationship between the adjacent ground objects more clearly. The thinking of the object-oriented change detection method is to analyze change before and after images based on texture features, and some characteristics of the feature space sort or multi-scale segmentation with image object and then the classification or segmentation of image object is used in the comparison to get the change information. The corresponding algorithms are multi-scale segmentation, interested target based and feature fusion based models [1-2].

To enhance the traditional pixel or the object based change detection algorithms, in this paper we incorporate the two methodologies and propose the novel model based on game theory analysis and hierarchical fuzzy clustering. The introduction of game theory will find the optimal balance of image segmentation and restoration to reserve the necessary features while eliminating noise. As the final 
step for generating detected change map we need to use the clustering model. However, the traditional pure fuzzy clustering takes the objective function of the static global control to discover the cluster distribution of data set which is just feasible for specific data sets. For optimization, we propose the revised hierarchical fuzzy clustering (HFC) model to achieve the better performance. The rest of the paper is organized as the follows. We firstly introduce the concept of game theory and apply it into the image segmentation and restoration and then we propose hierarchical fuzzy clustering theoretically to serve as the change map generating tool. Later, we finalize the systematic framework to undertake the change detection task. As the verification, we simulation the propose algorithm compared with other approaches to test the feasibility and robustness and draw the final conclusion.

\section{The Proposed Change Detection Algorithm}

\subsection{Game Theory and the Applications on Image Pre-processing.}

To better segment the images while keeping the necessary features contained in the images, we should find the balance of restoration and segmentation, the game theory could assist us to undertake the task. Game theory is for the multiple conflicts of the interest between stakeholders and mutual decision-making research and developed a discipline that is used to analyze human social behavior and management activities as well as the nature of biological behavior to each other. Evolutionary game theory is combine game theory with dynamic evolution process analysis of a new theory depict the game over time and the evolution of the relationship. Evolutionary game successfully explains the evolution process of some phenomenon and in the physics and other aspects have made remarkable achievements. The core issue of evolutionary game analysis is to determine its research methods or framework, namely game learning and strategy adjustment mode, or mechanisms. How to construct the dynamic mechanism to simulate the evolution game based on the specific situation in the game of learning and decision-making process is being popularly studied by domestic and foreign scholars [4].

Under this background, we choose to adopt Nash game theory to keep the best combination of image restoration and the segmentation process. Referring to the theoretical background, the game is made up of two objective functions denoted as $\delta_{1}$ and $\delta_{2}$. The control variables could be defined as $M$ according to theoretical derivation in [4]. The players in the game are 'segmentation' and 'restoration' and the restoration process could take in control of the intensity $\Phi$, segmentation operation could influence the discontinuity set defined as $\chi$. The player segmentation's target is to minimize formula one while the restoration's goal is to minimize to formula two.

$$
\begin{aligned}
& \delta_{2}=\sum_{i=1}^{K} \int_{\Omega}\left(\Phi-\Phi_{0}\right)^{2} d \vec{x}+v|\chi| \\
& \delta_{1}=\int_{\Omega}\left(\Phi-\Phi_{0}\right)^{2} d \vec{x}+\mu \int_{\Omega \backslash \chi}|\nabla \Phi|^{2} d \vec{x}
\end{aligned}
$$

Based on the literature [3] and [4], we propose the framework as the table one.

Table 1.The Proposed Game Theory based Image Pre-processing Framework

Algorithm. The Nash-game based Image Segmentation and Restoration Balance

1. To initialize the guess term: $S^{(0)}=\left(\Phi^{(0)}, \chi^{(0)}\right)$ and set the iterative control item $m=0$.

\section{Repeat.}

3. Set $\bar{\Phi}^{(m)}=\arg \min _{\Phi} \delta_{1}\left(\Phi, \chi^{m}\right)$ and $\bar{\chi}^{(m)}=\arg \min _{\chi} \delta_{2}\left(\Phi^{m}, \chi\right)$.

4. Set $S^{(m+1)}=\left(\Phi^{(m+1)}, \chi^{(m+1)}\right)$.

5. Set $m=m+1$.

6. Until $S^{(m)}$ obtains the optimal solution and converges. 


\subsection{Hierarchical Fuzzy Clustering.}

The basic idea of hierarchical fuzzy clustering algorithm is that $\mathrm{K}$ nearest neighbors chart describes the relationship between the strong similarities between objects. Due to different location of objects in the cluster have different fuzzy membership degree we should use the hierarchy method to obtain the cluster distribution status step by step. With the deepening of the hierarchy, distributed clusters inside tightness degree gets less, at the same level of processing conforms to close conditions in current layer with sub-manifold merge. The detailed steps of the algorithm could be summarized as the follows.

(1) Construct K-NN figure. K nearest neighbors figure is to describe the strong similarity relation between the object, because it only saves the object of k nearest neighbors. Object of neighborhood radius determined by the object area density, in densely populated areas, the neighborhood is defined while in sparse area, the neighborhood is defined. Neighborhood size of each object is adapted to their respective local area effectively, so it can describe objects dynamic neighborhood structure.

(2) Divide the figure. After getting nearest neighbors figure data set, with the proper graph partition algorithm to deal with it, thus forming the initial layer dense sub-manifold and lays the foundation for the subsequent hierarchical fuzzy clustering. The main characteristic of dense clusters is the internal connection is very strong, and more than connection between clusters, so we need to make sure that iterations of the algorithm are divided with the minimized weighted sum [2].

(3) Analysis of dense cluster type with clustering. With the analysis of the data characteristics and distribution of data set, each cluster can be stratified into the following three parts: the interior of the cluster near the center, clusters and other adjacent boundary part of the cluster, the internal and the boundary between the parts. The fuzzy degree of each is not identical in clusters of three parts, namely boundary ambiguity, almost completely belonging to the current clusters, cluster near the center and the middle part of the fuzzy degree between the former two.

\subsection{Optimized Image Change Detection Framework.}

Based on the prior discussion, our optimized change detection framework is constructed with the listed steps. (1) Generating the difference image. (2) Balancing the segmentation and restoration to form the better difference map for clustering. (3) Using the hierarchical fuzzy clustering algorithm to build up the change map. To reserve more features, we choose to use the fusion map as the difference image for analysis which is the combination of formula 3 and 4.

$$
\begin{aligned}
& X_{\text {sub }}(i, j)=\left|X_{1}-X_{2}\right| \\
& X_{\text {ratio }}=\left|X_{1} /\left(X_{2}+\varphi\right)\right|
\end{aligned}
$$

Where the $X_{1}$ and $X_{2}$ represent the images for processing, the $X_{\text {sub }}(i, j)$ is the manipulation of pure subtraction, the $X_{\text {ratio }}$ is the manipulation of division. In this paper, we use wavelet fusion proposed by Gong [5] to form the difference map defined as the equation 5.

$$
D_{\varepsilon}^{\text {Fusion }}(i, j)=\left\{\begin{array}{cc}
D_{\varepsilon}^{\text {sub }}(i, j), & E_{\varepsilon}^{\text {sub }}(i, j)<E_{\varepsilon}^{\text {ratio }}(i, j) \\
D_{\varepsilon}^{\text {ratio }}(i, j), & E_{\varepsilon}^{\text {sub }}(i, j) \geq E_{\varepsilon}^{\text {ratio }}(i, j)
\end{array}\right.
$$

\section{Experiment and Verification}

In this section, to validate the feasibility of our methodology, we conduct comparison simulation on both SAR images and the hyperspectral images. The figure one is the experiment on the image pre-processing with game theory framework where the (d) is the simulation without pre-processing and (c) is the ground truth for reference. The result is evident that the pre-processed method obtains better performance. In the figure 2 and 3, we demonstrate the comparison experiments on SAR and the hyperspectral images, respectively. (a) and (b) represent the images for analysis and (3) is ground truth image for reference. (d), (e), (f) are simulation results with the EM, K-NN and FCM algorithms. We could conclude from the results that our algorithm achieves better accuracy and robustness. As 
reflected form the red marked area, our methodology obtain better clustering performance while, at the same time, reserving the details and essential features.

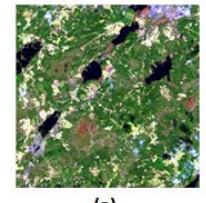

(a)

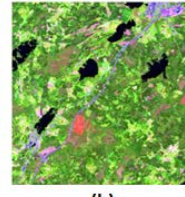

(b)

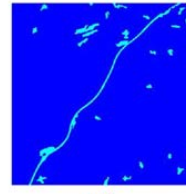

(c)

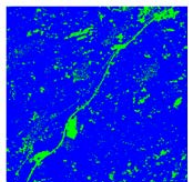

(d)

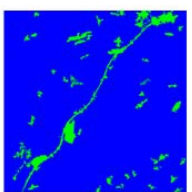

(e)

Fig. 1The Simulation on Image Pre-processing with Game Theory Framework
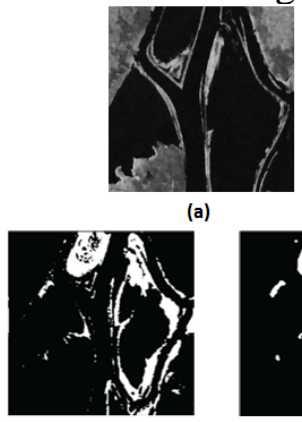

(d)

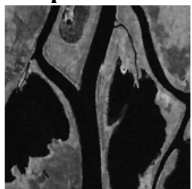

(b)
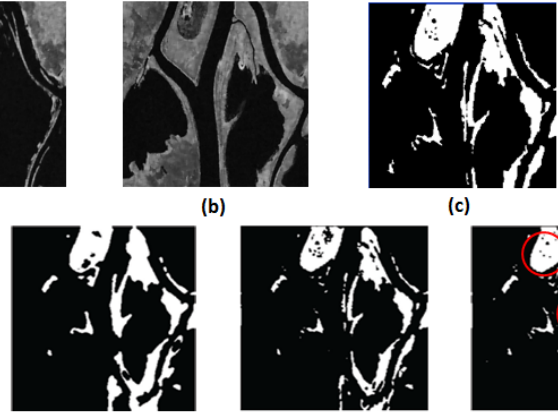

(e)

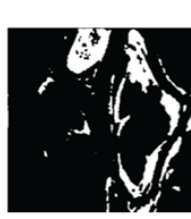

(c)

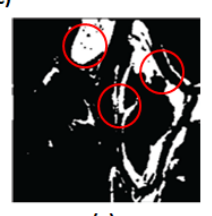

(g)

Fig. 2The Experiment on the Synthetic Aperture Radar (SAR) Images

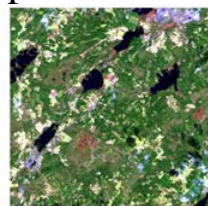

(a)

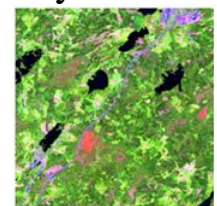

(b)

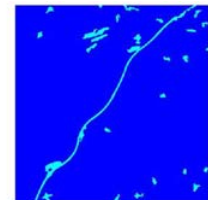

(c)

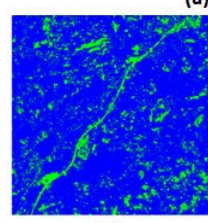

(d)

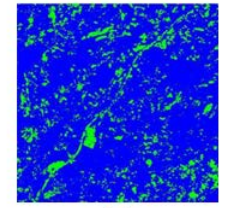

(e)

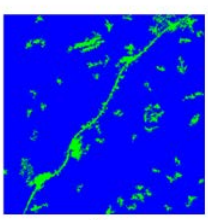

(f)

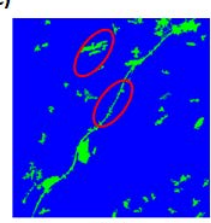

(g)

Fig. 3The Experiment on the Hyperspectral Images

\section{Summary and Conclusion}

In this paper, we have demonstrated the novel remote sensing image change detection algorithm based on game theory analysis and hierarchical fuzzy clustering. Firstly, to find the balance of image restoration and the segmentation, we employ the Nash game theory to find the tactical solution for the pre-processing and as reflected from literature review, the game theory could assist finding optimal choices. Later, to overcome drawbacks of traditional clustering approaches, we propose hierarchical fuzzy clustering to revise data clustering procedures. Then, we use the wavelet fused image to finalize the change detection framework. The experimental result shows that the proposed algorithm could integrate the advantages of restoration and segmentation to eventually achieve better performance.

\section{References}

[1]. Radke, Richard J., et al. "Image change detection algorithms: a systematic survey." Image Processing, IEEE Transactions on 14.3 (2005): 294-307.

[2]. Ding, Kun, et al. "Sparse hierarchical clustering for VHR image change detection." Geoscience and Remote Sensing Letters, IEEE 12.3 (2015): 577-581.

[3]. Bi, Chujian, et al. "SAR image restoration and change detection based on game theory." Intelligent Computing and Internet of Things, 2014 International Conference on. IEEE, (2015).

[4]. Kallel, Moez, et al. "A Nash-game approach to joint image restoration and segmentation." Applied Mathematical Modelling 38.11 (2014): 3038-3053. 
[5]. Gong M, Zhou Z, Ma J. Change detection in synthetic aperture radar images based on image fusion and fuzzy clustering. Image Processing, IEEE Transactions on. 2012 Apr;21(4):2141-51. 